\title{
BMJ Open Using decision trees to understand structure in missing data
}

\author{
Nicholas J Tierney, ${ }^{1,2}$ Fiona A Harden, ${ }^{3,4}$ Maurice J Harden, ${ }^{5}$ Kerrie L Mengersen ${ }^{1,2}$
}

To cite: Tierney NJ, Harden FA, Harden MJ, et al. Using decision trees to understand structure in missing data. BMJ Open 2015;5:e007450.

doi:10.1136/bmjopen-2014007450

- Prepublication history and additional material is available. To view please visit the journal (http://dx.doi.org/ 10.1136/bmjopen-2014007450).

Received 17 December 2014 Accepted 18 May 2015

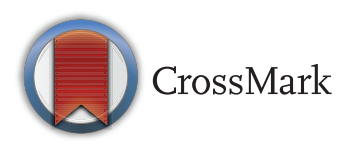

${ }^{1}$ Department of Statistical Science, Mathematical Sciences, Science \& Engineering Faculty, Queensland University of Technology, Brisbane, Queensland, Australia

${ }^{2}$ ARC Centre of Excellence for Mathematical and Statistical Frontiers (ACEMS), Brisbane, Queensland, Australia

${ }^{3}$ Faculty of Health, Clinical

Sciences, Queensland

University of Technology,

Brisbane, Queensland,

Australia

${ }^{4}$ Institute of Health and Biomedical Innovation, Brisbane, Queensland, Australia

${ }^{5}$ Hunter Industrial Medicine, Newcastle, New South Wales, Australia

Correspondence to Nicholas J Tierney; nicholas.tierney@qut.edu.au

\section{ABSTRACT}

Objectives: Demonstrate the application of decision trees-classification and regression trees (CARTs), and their cousins, boosted regression trees (BRTs) - to understand structure in missing data.

Setting: Data taken from employees at 3 different industrial sites in Australia.

Participants: 7915 observations were included. Materials and methods: The approach was evaluated using an occupational health data set comprising results of questionnaires, medical tests and environmental monitoring. Statistical methods included standard statistical tests and the 'rpart' and 'gbm' packages for CART and BRT analyses, respectively, from the statistical software ' $R$ '. A simulation study was conducted to explore the capability of decision tree models in describing data with missingness artificially introduced.

Results: CART and BRT models were effective in highlighting a missingness structure in the data, related to the type of data (medical or environmental), the site in which it was collected, the number of visits, and the presence of extreme values. The simulation study revealed that CART models were able to identify variables and values responsible for inducing missingness. There was greater variation in variable importance for unstructured as compared to structured missingness.

Discussion: Both CART and BRT models were effective in describing structural missingness in data. CART models may be preferred over BRT models for exploratory analysis of missing data, and selecting variables important for predicting missingness. BRT models can show how values of other variables influence missingness, which may prove useful for researchers.

Conclusions: Researchers are encouraged to use CART and BRT models to explore and understand missing data.

\section{BACKGROUND AND SIGNIFICANCE}

The motivating problem for this investigation was the analysis and reporting of occupational health data. The data set comprises 7915 observations of health variables reported on individual workers and corresponding environmental variables recorded at monitoring stations, at three worksites in Australia,

\section{Strengths and limitations of this study}

- This study demonstrates the utility in using decision tree statistical methods to identify variables and values related to missing data in a data set.

- This study does not address whether the missing data is missing completely at random (MCAR), missing at random (MAR) or missing not at random (MNAR).

observed from 2006 to 2013. Within each site, employees were grouped into Similar Exposure Groups (SEGs), based on the type of occupational exposure. For example, those working in administration are in the 'Support' SEG, and those who drive large construction vehicles are in the 'Production' SEG. Over the study timeframe, the number of medical visits per person ranged from 1 to 8. Health data included measures of lung function, body mass index (BMI), cholesterol, cardiac function and blood pressure, hearing, and psychological measures such as sleepiness, anxiety and depression. Environmental exposure data included measures of inhalable and respirable dust, and noise.

This data set is potentially rich in its ability to reveal relationships between health and environmental variables, differences in health profiles among SEGs, and health risk profiles for individual employees. However, there is a large amount of data missing in the data set, with approximately $63 \%$ of data missing overall. Here the proportion of missing data per row was calculated as the number of observed variables per row, divided by the total number of variables in a row. Consequently, prior to any analysis, it is important to understand the structure of this missingness and the potential impact that it might have on the analyses and resultant estimates.

A standard approach when seeing these data might be to run a linear regression of lung function being predicted by variables such as age, gender, SEG, smoking status and BMI. However, standard linear regression estimation methods require complete data, 
so cases with incomplete data are ignored, leading to bias when data is missing not at random (MNAR) or missing at random (MAR), and a loss of power when data are missing completely at random (MCAR). ${ }^{1-3}$ Although methods such as multiple imputation could be used to impute the missing values, care must be taken to avoid bias. ${ }^{2}$

Missing data are a pervasive feature of observational data. Three categories of missing data are usually identified. ${ }^{4}$ The first is MCAR, where missingness has no association with the observed or unobserved data. For example, assessments of lung function taken at a workplace may be missing for workers who are on vacation. If there is no known or measurable relationship between the timing of the tests and the timing of vacations, and if the other relevant features of the workers who are on vacation at the time of the tests are similar to that of other workers, then these missing data can be considered MCAR. The second category is MAR. This is a more specific case of MCAR where missingness depends on data observed, but not data unobserved. For example, if the missing lung function data occurs in workers who are being assessed for depression, and if there is no relationship between lung function and depression, then it can be considered as MAR. The third category is MNAR, where the missingness of the response is related to an unobserved value relevant to the assessment of interest. For example, if BMI is of interest, but those with especially large BMIs are more likely to have missing BMI data, these data can be considered as MNAR. It is important for researchers to recognise MNAR as it introduces bias into the estimation of associations and parameters of interest. For example, if lung function and BMI are negatively correlated, an estimate of BMI based on the MNAR may be too low.

These three varieties of missing data could be further divided into a knowable structure (MAR) or an unknown structure (MAR or MNAR), where the process driving data becoming missing are either known or unknown, ${ }^{5}$ and structure refers to variables and interactions that may influence missingness. Data MCAR are without a structure, as they are missing without any dependence on other variables. Determining whether this is known or unknown is important for determining whether bias may be introduced into the analysis.

\section{EXAMPLES OF MISSINGNESS}

Canonical sources of missing data are questionnaires. Data obtained from questionnaires are often subject to both unknown and known missingness structure. For example, MCAR data can arise from respondents accidentally failing to answer questions or inadvertently providing inappropriate answers. On the other hand, MAR data may arise due to the structure of the questionnaire. For example, the first question on a survey might be: 'If YES, skip to question 4', resulting in questions 2 and 3 missing. If the structure of the questionnaire is known, this type of missingness can be evaluated easily. However, if this information is not available, the mechanism responsible for producing missing data must be inferred from the data.

Another common source of known and unknown structured missingness is medical examination data. The results of particular medical tests may be: absent for purely random reasons (MCAR), due to the procedure (MAR), or based on decisions arising from the observed data (MNAR). For example, if a worker is young, they may not be subjected to neurodegenerative tests reserved for older workers, leading to MAR or MNAR data, depending on the aim of the analysis. A final example is dropouts in a longitudinal study, where participants do not return for future testing sessions. In this case, it is difficult, sometimes impossible, to ascertain the reason for the dropouts, and hence, whether the missingness is known or unknown, or MCAR, MAR or MNAR. However, this ascertainment is essential if the estimates based on these data are to be believed as unbiased. ${ }^{5-7}$

\section{EXISTING APPROACHES FOR HANDLING MISSING DATA}

Tests confirming whether data is MCAR or not are very useful as they open up the doors for the use of standard multiple imputation techniques. As described by Little, ${ }^{6}$ a standard approach to determine whether data are MCAR when only one variable, $y$, is missing from a data set is to compare those variables fully observed for responders and non-responders, using t tests to compare differences in means, or $\chi^{2}$ for differences in expected counts. Evidence against data MCAR is provided when a significant difference is observed. This approach can be extended to cases in which multiple variables have missing values, where the sample is split into cases with a given variable observed, or missing. Although this procedure is informative, it yields up to $p-1$ tests (where $p$ is the number of variables) for each variable and $\mathrm{p}(\mathrm{p}-1)$ statistics to assess the MCAR assumption. Inference on all these tests is problematic as the tests are correlated in a way that is dependent on the pattern of missing data and association of the y variables. This lack of independence affects the probability of type I errors (ie, erroneous declaration of statistics significance), and makes it difficult to gain clear inference on the nature of missingness, as illustrated in our Results section.

To combat this problematic process, Little proposed a single test statistic for testing MCAR. This involved an evaluation of equality of means between identified missing data groups. Rejection of this test result gives strong evidence that the data are not MCAR. Little's test of MCAR is widely used today, especially in social science $^{8}$ and medical research. ${ }^{9}$

Recent research has also provided statistical tests and software that evaluate missing data via patterns, equality of means, and homogeneity of variance, and allow for non-normal data. This is achieved, for example, in the 
MissMech package for the R statistical software, ${ }^{10}$ which uses imputation (from either normal or non-normal distributions) to compare means and covariances. These tests enable the researcher to determine whether or not there is sufficient evidence for data to be declared as MCAR. However, understanding how and why missingness is being generated can become arduous when handling larger data sets, as they can have many missingness patterns, making inference difficult for the same reasons as having $\mathrm{p}$ variables and $\mathrm{p}(\mathrm{p}-1)$ statistics, as explained previously.

In addition, reliance on statistical significance testing to assess whether data are missing may fail to address settings where there may not be significant missingness, but a complete case analysis may still result in bias. ${ }^{11}$ Approaches for better understanding missingness that are simple to understand and implement, are therefore still in demand.

Common methods of handling missing data, such as complete case analysis, missing indicator method, and last case carried forward have been shown to be acceptable when data is MCAR. ${ }^{12}{ }^{13}$ That being said, most recommendations now are to use multiple imputation, but subject to some care as it only reduces bias from analysis when data are MAR or MCAR; multiple imputation also requires variables that influence missingness to be included in the imputation model. ${ }^{1-4}{ }^{14}$ When data are MNAR, multiple imputation can be used but requires the MNAR mechanism to be known, which is not often undertaken in practice. ${ }^{3}$ Improving the understanding of missingness structure in a data set allows for consideration of other appropriate multiple imputation methods, or other methods to incorporate partially observed variables, such as random effect models, Bayesian methods, down-weighting analyses, or pattern mixture models. ${ }^{2} 1516$

There are various approaches and packages specifically developed to explore missing data, and resultant imputation methods. These include: $\mathrm{R}$ packages VIM, Amelia, mi, the MANET program,${ }^{17}$ as well as the standalone software-MissingDataGUI. ${ }^{18-21}$ These packages facilitate the graphical exploration of data prior to and after imputation to evaluate missingness trends and causations, and imputation accuracy, respectively. These methods require the user to visually search for and find missingness trends, and infer interesting structure. ${ }^{17} 22$ While humans are very good at finding patterns, a model-driven approach provides a more precise and potentially more automatic framework for exploring missing data. We propose the use of decision trees as a complementary tool for doing this.

\section{OBJECTIVE}

Decision trees, in particular, classification and regression trees (CARTs), and their cousins, boosted regression trees (BRTs), are well known statistical non-parametric techniques for detecting structure in data. ${ }^{23}$ Decision tree models are developed by iteratively determining those variables and their values that split the data into two groups, so that the response is most homogeneous within the groups, and there is greatest difference between the groups. ${ }^{23-26}$ This paper demonstrates the application of CARTs and BRTs in understanding the structure of missing data.

\section{MATERIALS AND METHODS}

Decision tree models are typically represented as treelike structures. A CART analysis typically returns a single tree with multiple splits, depicted as multiple branches. Growing a tree involves recursively partitioning the response into two parts based on some value of a variable that best splits the data. The variable and split point are chosen to optimise a given goodness-of-fit criterion, such as minimising the residual sum of squares for continuous data, or a measure of node purity (eg, gini index or cross-entropy) for categorical data. ${ }^{23}$ This recursive partitioning continues until a selected stopping rule is reached, such as when there are fewer than 10 observations in each final partition-terminal node. ${ }^{24} 27$

The final depth of the tree, the tree complexity, is measured by the total number of splits determined by various goodness-of-fit measures designed to trade-off accuracy of estimation and parsimony. A large CART model can be grown to fit the data very well, leading to overfitting and a reduced capability to accurately fit new data (robustness). To improve robustness in CART models, one can use cross-validation and cost-complexity pruning, where models are grown on subsets of the data and then some 'best' model is selected using criterion that best reduce a cost-complexity parameter. ${ }^{24} 252728$

A useful feature of decision trees is the way that they handle missing data. Whereas some methods, such as linear regression, often default to only using complete data to predict an outcome, decision trees use the surrogate split method. This means that when a value for a variable is missing, and that variable needs to be used to determine a split, an alternative variable that is highly correlated with the missing variable is used to determine the direction of the split. ${ }^{24}$

In contrast to CART, a BRT analysis typically generates many sequentially grown simple trees based on random samples of the data. Each sequentially grown tree focuses on the errors of the previous tree, resulting in a model where emphasis is placed on observations that are poorly modelled by the existing collection of trees. The boosted model returns a list of the variables used to create the splits in the different trees. A 'relative weight' is then calculated for each variable by taking the average number of times a variable is chosen for splitting weighted by the squared improvement to the model from each split and scaled to sum up to $100 .^{29}$ Larger weights indicate stronger influence.

Boosted regression trees require the parameters learning rate and tree complexity. It is worth noting that 
these terms are also referred to as shrinkage parameter and tree complexity, respectively. The learning rate controls how much each tree contributes to the model as it develops. Typically, a smaller learning rate provides better prediction than a larger learning rate. The tree complexity sets the number of interactions fitted in the model, where a tree complexity of two allows for two-way interactions, three allows for three-way interactions, and so on. ${ }^{26}$ Creating reproducible results in the BRT model requires setting a random seed, as the process used to create the BRT model involves random subsampling of data.

Whereas the single trees produced by the CART analysis are appealing, they are less able to predict linear relationships, are very sensitive to small variations in data, and may provide an oversimplification of the 'real' model. ${ }^{30}$ In contrast, the BRT analysis is better able to describe linear relationships and is more robust in terms of predictive accuracy, although interpretability suffers as a result. ${ }^{26}$ Using both CART and BRT models provides complementary inference-one is simple but provides interpretability, the other provides complexity and robustness, but with reduced interpretability.

CART and BRT models were applied to the case study data, using per cent data missing per row as the response variable and the following explanatory variables: Site, UIN (unique identifying number), Sex, Type (of data), Date, FVC, FVC\%, FEV1, FEV1\%, FEV1\%, FVC\%, SEG Primary, Age, BMI, Code, Systolic Blood Pressure, Diastolic Blood Pressure, HDL Cholesterol, Total Cholesterol, Cardiac Risk Score, Smoking, Epworth Sleeping Scale, Secondary SEG, K10 Depression, ETOH Alcohol Scale, BHL, Repeated Visits, Exercise Per Week, Weight, Height, Waist, Blood Sugar Level, Pulse, Concentration, LAeq. These variables can be seen in table form in online supplementary table S1.

The statistical software package ' $R$ ' and the graphical user interface, 'RStudio' were employed for the analyses. ${ }^{31}{ }^{32} \mathrm{R}$ packages 'rpart' and 'gbm' were used for the CART and BRT analyses. ${ }^{27} 33$ The rpart model handles missing values by using surrogate splits: when a value for a variable is missing, and that variable needs to be used for a split, an alternative variable with a similar splitting property is used to determine the direction of the split. The gbm function also uses a surrogate split method.

The current analysis generated CART models using the default values specified in 'rpart ${ }^{\text {'27 }}$ and BRT models using the guidelines provided by Reference 26, which build on the package 'gbm'. ${ }^{33}$ The BRT model was run assuming a Gaussian error distribution for the response, an interaction depth of 5 , learning rate of 0.01 , and bagging (fraction of training set observations randomly selected) set to 0.5 .

When there is extensive missing data, those variables identified as important for describing missingness structure may also be missing. This was observed in the case study, and may affect the reliability and/or validity of results and predictions. To explore how missingness may affect the CART and BRT models, a simulation study was conducted, such that CART and BRT models were applied to smaller data sets with missing data inserted artificially. These are described following the results of the case study analysis.

As noted earlier, the case study contained a very large amount of missing data. The overall proportion missing was 0.63 . The missingness map (from the $\mathrm{R}$ package 'Amelia', ${ }^{19}$ shown in figure 1 , displays whether data is missing (grey) or present (black), for each case.

\section{RESULTS}

As an exploratory assessment to determine whether there was sufficient missingness to warrant an investigation, $\mathrm{t}$ tests and $\chi^{2}$ tests were used to assess whether the presence or absence of BMI, FEV1, FVC, FEV1/FVC, and concentration, influenced either the mean values of other variables (via a t test), or the expected count of a particular factor (via a $\chi^{2}$ test). Results indicated that consistent sets of variables were affected, suggesting a potential pattern or structure of missingness. Those variables affected are listed in table 1 . These variables, and their mean values or expected counts, were reported to the industry collaborator to help explore the causes of missing data and consider down-weighting them in other analyses.

The CART and BRT models were run as described in the Materials and methods section. The CART model obtained from the analysis of the case study data is represented in figure 2. The tree indicates that the type of data best predicts the proportion of missing data in an individual's record. There are three main classes of data type: medical (Type 1), follow-up medical (Type 2), and hygiene or environmental exposure (Types 3-6). The missingness proportion for each type can be seen in the violin plot in online supplementary figure S1. The prediction from the CART model is such that when Type is 1 (medical data), there is a lower proportion of missing data $(30 \%)$, compared with the right split, when data are of Type $=2-6$, (repeated medical and environmental exposure; $74 \%$ missing data). Another split occurs within Type 1, where data from site 3 has less missing data (22\%) compared to sites 1 and 2 (34\%). Another split occurs based on Type 2 (repeated medical data) compared to Types 3-6 (environmental exposure), where data of Type 2 has $64 \%$ missing data, and data of Types $3-6$ has $76 \%$ missing data. Within Type 2, there is a split for repeated visit, such that for those with one visit, there is $37 \%$ missing data, and for all other visits (2-8) there is $65 \%$ missing data.

The analysis ably demonstrated the utility of this modelling approach in identifying those variables and their values that are important for predicting missingness structure. From this model, we were able to confer with data collectors to determine that the 'Types' of data were originally separate data sets, which were then 
Figure 1 Missingness map showing the amount of missing data in the case study. The horizontal axis indicates the variables in the data set, and each individual in the study is a row in the y axis. Black indicates present data, grey indicates absent data.
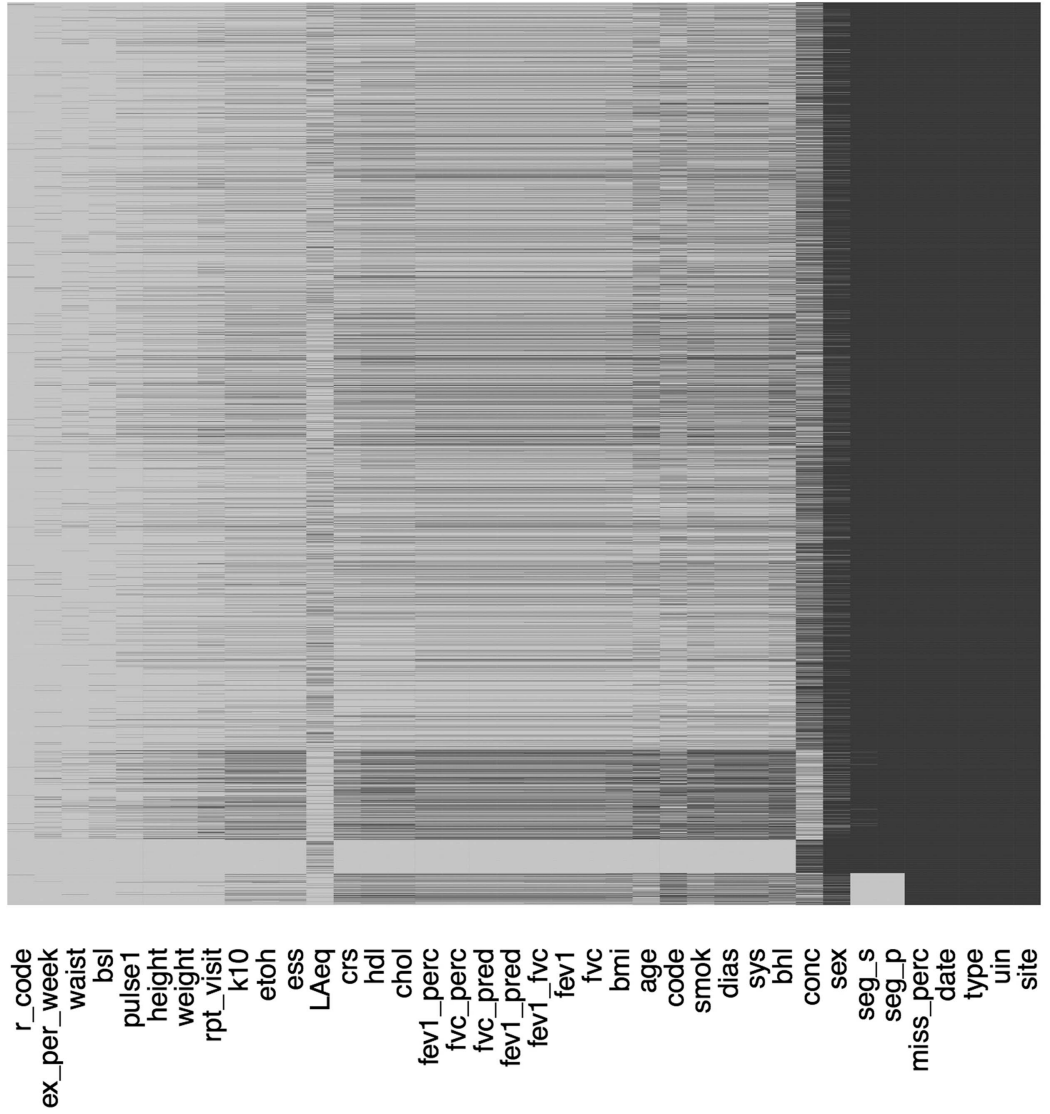

combined and represented as records for each individual (employee), resulting in many missing values per record. We were also able to identify that different

Table 1 Variables affected by presence/absence of BMI, FEV1, FVC, FEV1/FVC and concentration

\begin{tabular}{|c|c|}
\hline $\begin{array}{l}\text { Presence/ } \\
\text { absence of }\end{array}$ & Variables affected \\
\hline BMI & $\begin{array}{l}\text { Date, Age, SYS, DIAS, HDL, CRS, } \\
\text { BHL, Missing\%, FEV1/FVC, FEV1\%, } \\
\text { Site, Type, SEG (P), Code, SEG (S), } \\
\text { Rpt Visit, Smoking, Sex }\end{array}$ \\
\hline $\begin{array}{l}\text { FEV1, FVC, } \\
\text { FEV1/FVC }\end{array}$ & $\begin{array}{l}\text { Date, Age, SYS, DIAS, HDL, CRS, } \\
\text { BHL, Missing\%, FEV1/FVC, FEV1\%, } \\
\text { Site, Type, SEG (P), Code, SEG (S), } \\
\text { Rpt Visit, Smoking, Sex, Ex/week }\end{array}$ \\
\hline Concentration & $\begin{array}{l}\text { UIN, Date, Missing\%, Site, Type, } \\
\text { SEG (P), SEG (S) }\end{array}$ \\
\hline \multicolumn{2}{|c|}{$\begin{array}{l}\text { Age, age at time of examination; BHL, binaural hearing loss (\%); } \\
\text { BMI, body mass index; Code, medical code; CRS, cardiac risk } \\
\text { score; Date, date of examination; Dias, diastolic blood pressure; } \\
\text { Ex/week, \# planned exercise sessions per week; FEV1/FVC, ratio } \\
\text { of FEV1\% to FVC\% (FVC, forced vital capacity; FEV1\%, forced } \\
\text { expiratory volume in } 1 \mathrm{~s} \text {; HDL, high density lipoprotein cholesterol; } \\
\text { Missing \%, the per cent of missing data in that row; Rpt Visit, } \\
\text { number of medical attendances; SEG(P), primary SEG; SEG(S) is } \\
\text { the secondary SEG; Sex, gender; Site, site the data belongs to; } \\
\text { Smoking, smoking status of employees-current, ex, or } \\
\text { non-smoker; Sys, systolic blood pressure; Type, type of data } \\
\text { (1=medical, } 2=\text { =follow-up medical, 3=inhalable data; } 4=\text { respirable } \\
\text { data; } 5=\text { silica exposure data; } 6=\text { noise exposure data); UIN, unique } \\
\text { identifying number for an employee. }\end{array}$} \\
\hline
\end{tabular}

variables were measured at sites 1 and 2 compared to site 3 , and that repeated measures had less data as tests became more specific for subsequent visits.

Figure 3 provides a graphical evaluation of model fit of the CART and BRT models. Figure 3A shows the predicted proportions of missing data per row based on the

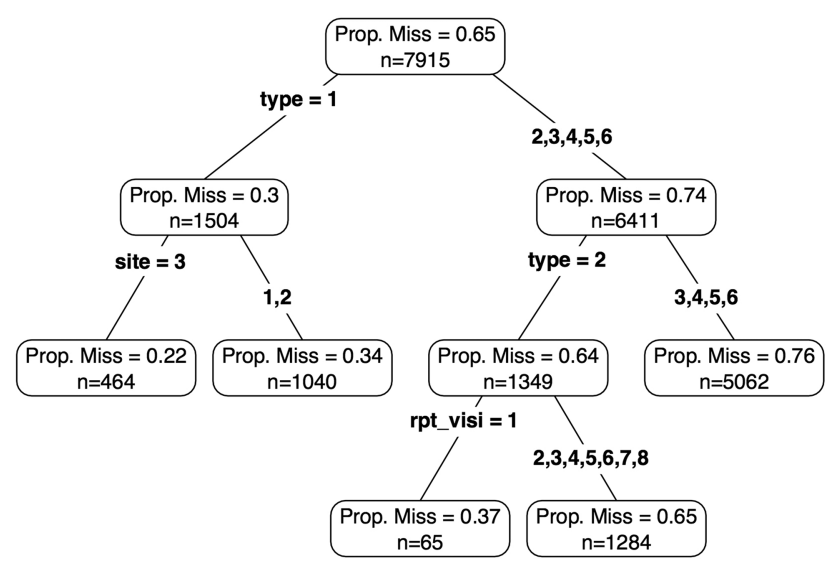

Figure 2 CART analysis of the case study data, indicating that type of data and repeated visit (rpt-visit) are important predictors of the proportion of data missing. The three numbers in each oval indicate the expected proportion of missing data (Prop. Miss) per row of data (ie, individual's record) and the number of rows $(n)$. Definitions of variables used for splits are given in the caption of table 1 (CART, classification and regression trees; BRT, boosted regression tree). 
Figure 3 Comparison of

observed (horizontal axis) and of data missing per row, based on (A) the CART model (top left) and (B) the BRT model (top right). All points in these plots have a small jitter added to their position so that repeated points can be seen. The bottom panel $(\mathrm{C})$ also also shows the error distribution of the BART and CART results, with both having good prediction (close to 0), and the CART model having a wider distribution (BRT, boosted regression tree; CART, classification and regression tree). predicted (vertical axis) proportion
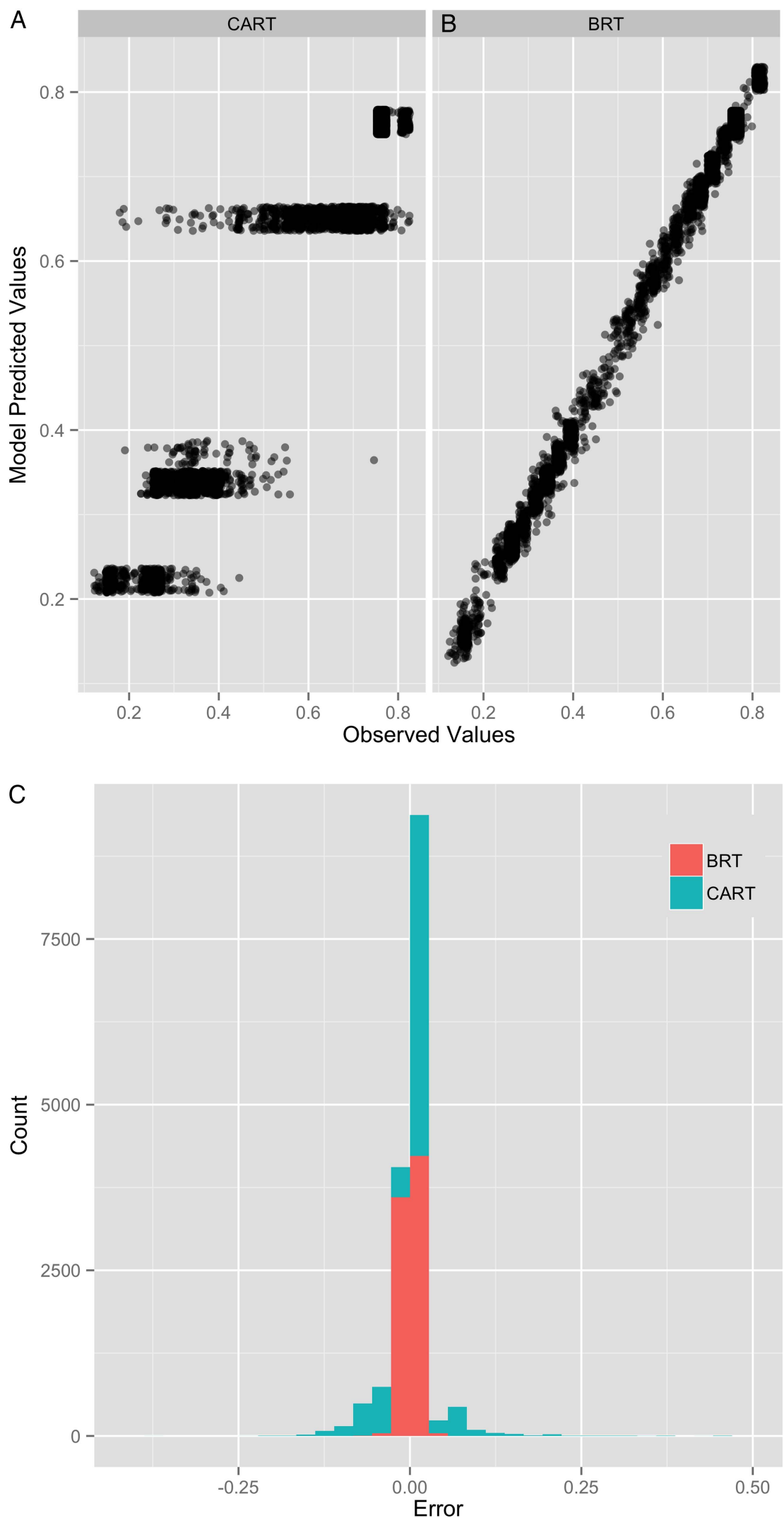

CART model, compared with the observed proportions. It is apparent that the model can accurately predict small and large proportions of missing data, but is less accurate at predicting moderate proportions. This predictive resolution is a result of the trade-off between robustness, parsimony, and accuracy, reflected by the degree of pruning of the tree. Allowing more branches in the model on the right panel in figure 3 provided a better fit to the observed data but may lead to overfitting. The predictive resolution was also a result of using 
a single tree rather than multiple trees, ${ }^{30}$ motivating the complementary use of BRTs. The comparison of predicted and observed values of proportion of missing data from the BRT model in figure $3 \mathrm{~B}$ confirmed that this model provides improved goodness-of-fit. Figure 3C also shows that the CART and BRT models provided mostly very accurate model fits, with the BRT model having a comparatively tighter error distribution compared to the wider distribution of the CART model.

Results from the BRT model also give the relative importance of variables in predicting the proportion of missing data; figure 4. This analysis shows that obesity (measured by BMI) and lung function (measured by FEV1 and FVC) are the most important variables for prediction of missingness.

Figure 5 shows the observed proportions of missingness compared with the fitted function based on the BRT model, for the first nine variables indicated in figure 4 . The centre of the vertical axis indicates the model expected proportion of missingness. As might be anticipated, those variables with more definite nonlinearity in the fitted function have more influence in the BRT analysis. For example, more missingness is anticipated in individuals with higher BMI or lower lung function measurements.

\section{SIMULATION STUDY}

Two experiments were created to explore the capability of decision tree models in elucidating the induced missingness structure.

\section{Experiment one}

In this first experiment, data sets were created where the variable instigating the missingness was either (1) not missing, or (2) 50\% MCAR. These new data sets contained five variables, two categorical and three continuous, with 1000 observations in each. The two categorical factors, F1 and F2, ranged uniformly across categories nominally labelled $1-7$, and $1-10$, respectively. The three continuous variables, C1, C2 and C3, were normally distributed with means and SDs of 50 and 10, 90 and 10 , and 30 and 3 , respectively.

These variables and values were chosen to represent specific variables in our data set. C1: age; C2: lung function; C3: BMI; F1: SEGs; and F2: a score obtained from a measurement. The variable $\mathrm{C} 1$ determined whether C2, C3, F1 and F2 were missing, such that when C1 was greater than 55 these variables went missing with probability 0.95 . C1 was selected as the missingness instigator to mimic a scenario where someone aged 55 years is not measured on a variety of variables.

The CART and BRT models were assessed on 100 simulated data sets for each of these two scenarios, where the outcome is the proportion of missing data in the variables C1, C2, C3, F1 and F2.

Model performance in the first experiment was evaluated based on the following criteria:

- Did the model predict the variable, C1, as responsible for the missingness?

- Did the model identify the threshold value of 55 for the variable $\mathrm{Cl}$ as the value causing the missingness?
Figure 4 Relative importance (RI) of variables in predicting the proportion of missing data per row based on a BRT analysis. Only variables with $\mathrm{RI}>1$ are the variables included, in order of importance (left to right) are BMI (25.57), FEV1 (25.25), FEV1 (Predicted) (14.22), FVC (11.34), FVC (Predicted) (6.266), Type (4.23), FEV1 (Percent) (1.80), Smoking (1.66), Systolic Blood Pressure (1.58), Blood Sugar Level (1.02), K10 Depression score (1.00) (BMI, body mass index; BRT, boosted regression trees; FEV1, forced expiratory volume in $1 \mathrm{~s}$; FVC, forced vital capacity).

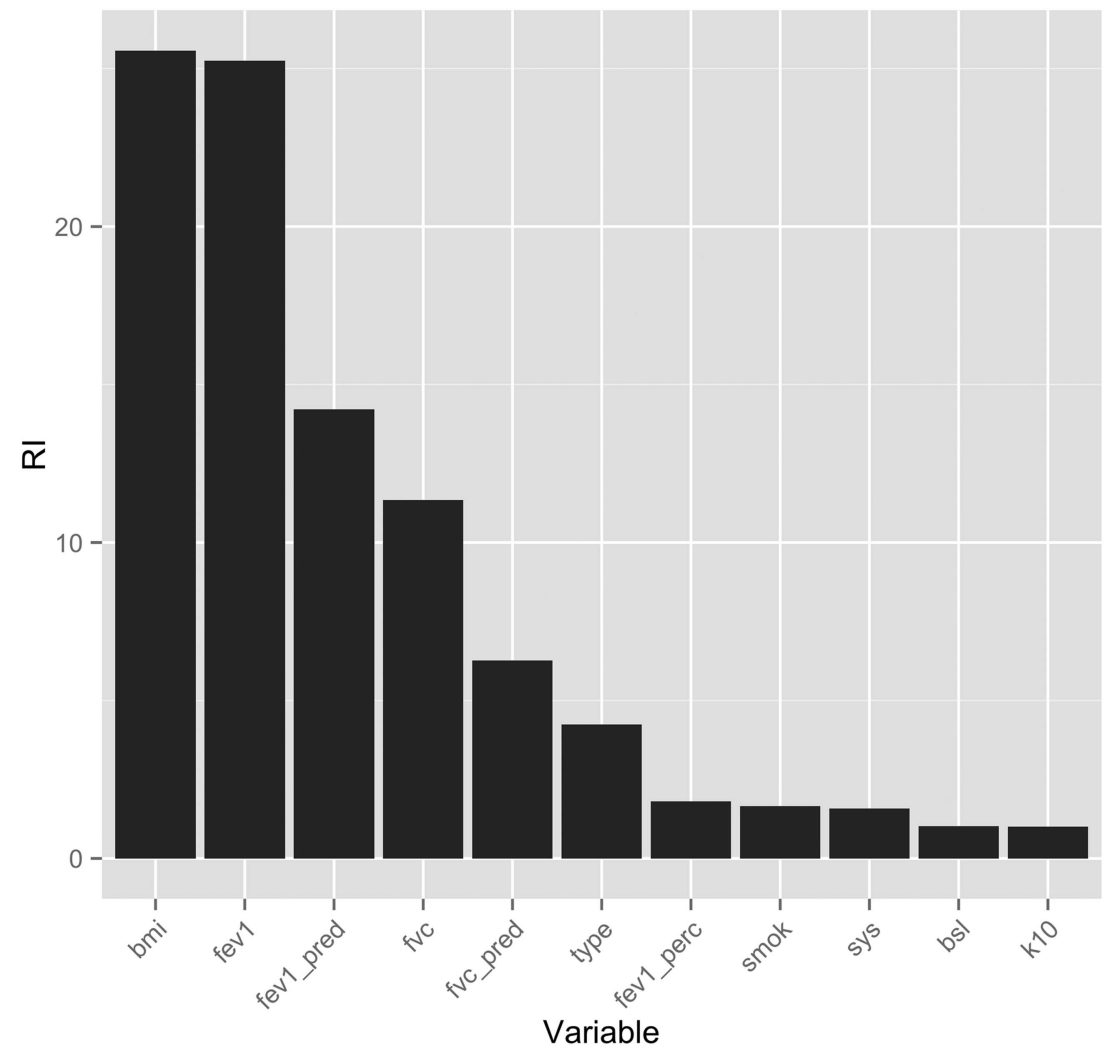



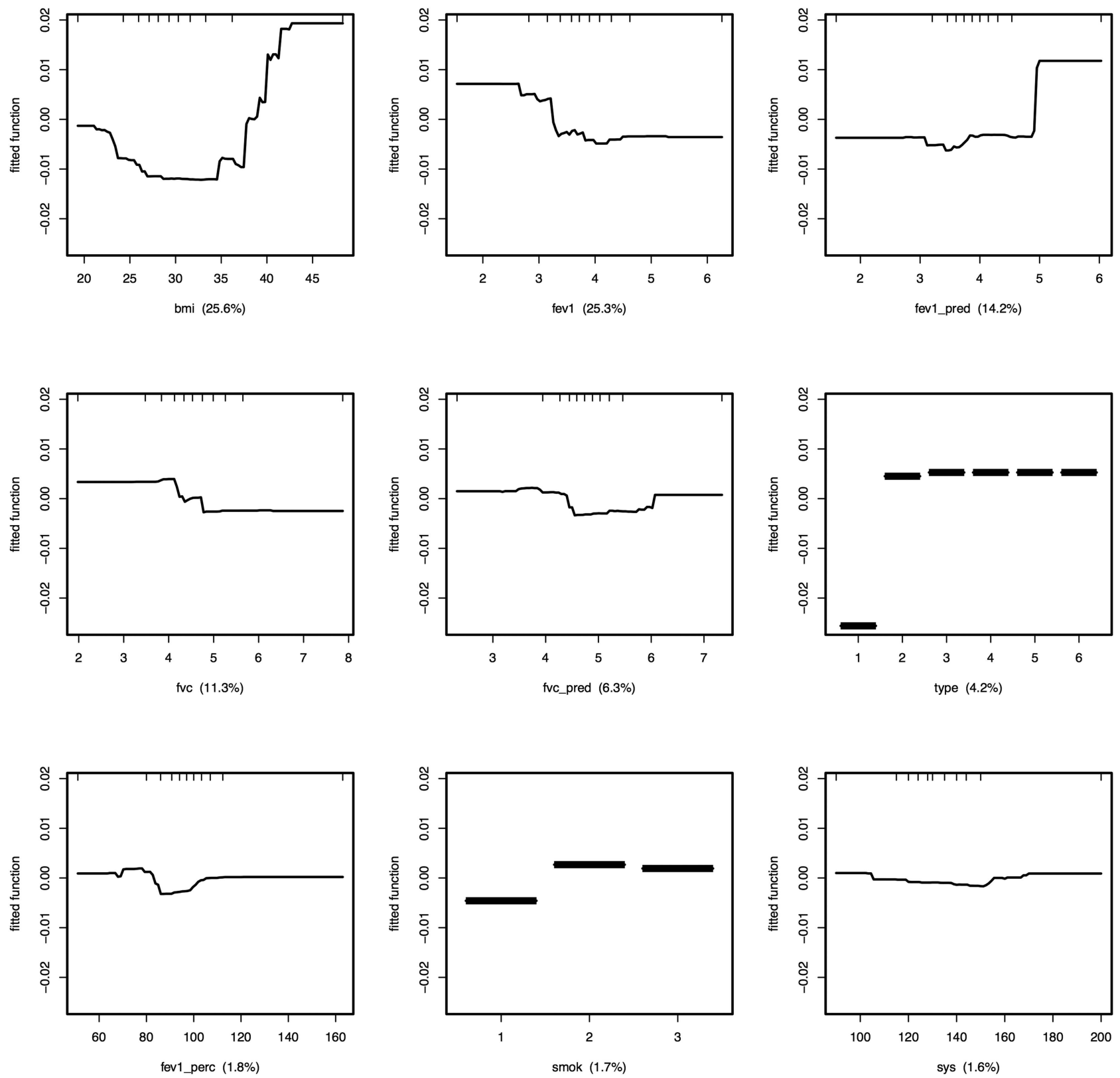

Figure 5 Fitted function of variables based on the boosted regression trees model with the zero-point of the vertical axis indicating the model expected proportion of missingness. Lines above 0.00 indicate more missingness than expected, and lines below indicate less missingness. Note that type and smoking (smok) are represented differently as they are discrete, whereas the remainder are continuous.

If the models performed well in this first experiment, we have confidence that the models can identify structured missingness.

\section{Experiment 2}

The second experiment explored the performance of decision trees for use with MCAR data. For the second experiment, the CART and BRT models were assessed on two data sets, MCAR 20\%, or MCAR 50\%, with 100 simulated data sets created. In this experiment, the simulated data sets were the same as the first experiment with the addition of two variables, R1 and R2, drawn from a random uniform distribution. These last two variables were deliberately included as 'noise' in the simulations to assist in assessing whether the models are overfitting the data. In addition to the criteria used for experiment 1, we assessed experiment 2 based on the variance in the measures of variable importance as we are interested in exploring whether these variables are consistently selected as important in an MCAR scenario. If this is the case, then we can assume the decision tree models are simply picking up on noise, rather than signal. These variables represent a small, simple and realistic data set that we would encounter at our industry site (except for variables R1 and R2 from experiment 2). The intention was to evaluate the missingness 
represented by our real data set, MAR and MNAR, and compare it to data MCAR to evaluate model performance.

Variable importance was measured for each of the simulated data sets, and was compared with the case study data set. For both experiments in the simulation study, the BRT model had a smaller interaction depth of 2 , rather than 5 that was used in the case study analysis, as the simulation study data set had far fewer variables.

\section{Simulation study results}

In the first experiment, for both parts 1i (not missing) and 1ii (50\% MCAR), the CART model identified that the variable $\mathrm{C} 1$ was responsible for instigating the missingness, satisfying criterion A. The CART model also correctly identified the split for $\mathrm{C} 1$, such that when the threshold C1 $>55$ there was an increased amount of missingness, and this satisfied criterion B. All developed CART models selected $\mathrm{C} 1$ for the split and the value 55 , meaning that all models were essentially identical. These models can be viewed in online supplementary figure S2.

Intriguingly, the BRT model was unable to identify $\mathrm{C} 1$ as the most important variable in predicting the proportion of missing data, irrespective of whether $\mathrm{Cl}$ was not missing, or 50\% MCAR. Hence the BRT model did not satisfy criterion A. However, when inspecting model predictions against variable values, the BRT model predicted a change in missingness as $\mathrm{C} 1$ reached 55. These fitted functions can be seen in online supplementary figure S3. This BRT model satisfied criterion B.

For the BRT model, there was variation around variable importance in this simulation study, such that when there was more missingness, there was greater variation in variable importance. An illustration of this is given in online supplementary figure S4. The CART model always used $\mathrm{C} 1$, and the value 55 to split on, and so evaluating variable importance is somewhat irrelevant.

For the second experiment, the data were either (1) $20 \%$ MCAR, or (2) 50\% MCAR. The CART model showed different levels of variable importance over simulated data sets, and that the spurious random variables R1 and R2 were often identified as important. This can be seen in online supplementary figure 4 .

The variation in variable importance was smaller in the resampled case study data set, compared with experiment 2. The BRT model, such as the CART model, also chose variables R1 and R2 as relatively important in predicting missingness. Visual depictions of the variation in variable importance for the CART and BRT models over the experiment and case study data can be found in online supplementary figures 6-8.

The difference in variation of variable importance for simulated versus the resampled case study data provided evidence that data MCAR produces greater variation in variable importance. There was less variation in variable importance for simulated data compared to case study data, suggesting the case study data does have a missingness structure.

\section{DISCUSSION}

In this paper, we proposed the use of decision tree models, notably CARTs and BRTs, for inspecting the structure of missingness in observational data. To the authors' knowledge, this is the first time that these decision tree models have been proposed for this purpose. The application of the models to a substantive case study involving occupational health data, specifically medical tests for employees, demonstrated the complementarity of the analyses. Whereas the CART model identified three variables: type of medical; how many visits the employee had and site; the BRT model identified BMI and lung function as the most important factors predicting the proportion of missingness in the employees' health records. In addition, the BRT analysis also modelled the expected missingness for variable values.

The case study partners found that these results revealed important known and unknown structure in the data. An example of structure that was known to exist but not known to have such dominant influence, was that the data set was a collection of smaller databases coming from different sources, denoted by the values of type; that is, Types 1 and 2 are different kinds of medical data, and Types 3-6, environmental exposure data. The data sets were originally combined in this fashion so that data could be matched by ID number, allowing deidentified inspection of individual results. Where matching was not possible, group results could be observed. As a result of this concatenation of different data types, large chunks of data were missing, as the sources collected different kinds of information and used different IDs, preventing data matching. Further exploration of the relationship between missingness and type revealed that the majority of missing data was missing for Types 3-6, compared to Types 1 and 2. This is demonstrated in the violin plot in online supplementary figure $\mathrm{S} 1$.

Another missing data structure revealed in our analyses was found by comparing results from the CART and BRT analyses. The focus of the CART analysis was on type, site and repeated visits. Compared with the CART model, the BRT analysis focused more deeply on the medical data, and highlighted that extreme values for variables such as BMI or lung function, had more missing data. Discussion with industry partners on these findings revealed that individuals with extreme values for measurements such as BMI or lung function require follow-up tests. As follow-up tests are taken on a small specific set of variables relating to the particular health query or concern, they result in more missing data in the overall data set. Discovering these missing data structures has resulted in future research being conducted on subsets of data with selection based on these missing data structures. This allows for more representative, 
reliable and valid results. It may also motivate different and more informed methods of data analysis and modelling.

In our analysis, we used the proportion of missing data in a row as our response. This has the advantage of accommodating correlation between variables and providing a single, easily understood, summary statistic for missingness. Alternative measures of missingness of the data set could be used, such as missingness in individual variables, or an index based on a factor analysis, or similar dimension reduction method. These could then be used to predict other structural features of the data, such as multiple individual variable's missingness in a multivariate analysis, or clusters of missingness, and would tell us different things about the missingness structures in the data set.

The analysis of missing data described in this paper is not limited to decision trees, and could be extended to other analyses such as neural networks, random forests, and Bayesian Learning Networks. Moreover, decision trees themselves can be implemented using different variable selection methods, although recursive partitioning is the standard choice. ${ }^{24}{ }^{27}$ As illustrated in this paper, decision trees using recursive partitioning were desirable for ease of implementation, handling non-parametric data, and automatic handling of missing data.

It was mentioned in the introduction that knowing the structure of the missing data may not give a clear indication of the mechanism (in terms of MCAR, MAR, MNAR). However, understanding the missingness structure can help lead the researcher to create better imputation models or use alternative methods of addressing missing data, as well as improve future data collection or conduct their own further investigations into missingness structure. $^{3}$

Our simulation analysis performed the decision tree analysis on MCAR and MAR scenarios to evaluate model performance using a simple, known example of missingness. In the case study, however, although MAR and MCAR variables are present, the dominant form of missingness is MNAR due to the nature of the medical examinations. Thus, the methods suggested in this paper have been demonstrated to be effective for all three types of missingness. However, as indicated in the introduction, MNAR scenarios could be envisaged whereby the data explaining the missinginess are not observed structurally. This motivates further research on this issue.

\section{CONCLUSION}

The use CART and BRT models have allowed us to develop our understanding of missingness structure in the data. The authors' experience in using these models was that they motivated the appropriate questions to explore the missing data structure, leading to a better understanding of the origins of the data. This understanding will help improve both data collection and the handling of missing data in future analyses.
The results of the simulation study were surprising. Despite the a priori expectation, based on published literature that the BRT model would be more robust and accurate than the CART model, this was not borne out in the analysis. The BRT model accurately predicted whether or not there was substantial missing data, and the diagnostic charts provided a visual indication of how missingness behaves for variables. However, in the simulation study, BRT was unable to select the correct variable as the most important for predicting the (known, modelled) missingness structure in the data. In contrast, the CART model did this consistently.

Experiment 2 involved the evaluation of decision tree performance on data MCAR (20\% or 50\%) using the simulated data sets from the first experiment with the addition of two variables, R1 and R2, drawn from a random uniform distribution. R1 and R2 were included in these simulations to assist in exploring which variables were important for splitting in the CART and BRT models when there was no structure in the missingness. Results from experiment two demonstrated that both the CART and BRT models had greater variation in variable importance when more missingness was introduced, although this seemed to relate to the degree that the dependent variable was missing.

Although this study has demonstrated the utility in using decision tree statistical methods to identify variables and values related to missing data in a data set, it is noted that these methods do not address whether the data is MCAR, MAR or MNAR, and they do not specifically outline the bias that is in the data due to missingness. Instead, these methods are helpful in determining why and how data are missing. It is still up to the researcher to understand the potential bias that this may or may not cause.

Twitter Follow Nicholas Tierney at @TierneyNicholas

Acknowledgements The authors thank Dr Nicole White and Dr Jegar Pitchforth for assistance and helpful discussions. The authors also thank the reviewers for their constructive comments.

Contributors NJT had the initial idea to explore missing data using decision trees, performed all analyses, and wrote the first draft. KLM provided guidance in selecting and interpreting analyses, designing the simulation study, and critical review of manuscript. FAH and MJH assisted in the acquisition of data, interpretation of results from an industry perspective, and critical review of manuscript. All authors approved the version of the paper for publishing, and agreed to respond to questions that may arise regarding integrity of the work.

Funding This research was jointly funded by an Australian Postgraduate Award (APA), the Australian Technology Network Industry Doctoral Training Centre (IDTC), Hunter Industrial Medicine, the Australian Research Council, and the ARC Centre of Excellence for Mathematical and Statistical Frontiers.

Competing interests None declared.

Ethics approval The QUT University Human Research Ethics Committee.

Provenance and peer review Not commissioned; externally peer reviewed.

Data sharing statement Statistical code and simulation data sets are available from the corresponding author at Dryad Repository, who will prove a permanent, citable and open access home for the materials. This can be accessed via the Dryad repository at http://datadryad.org/ with the doi:10.5061/dryad.j4f19. 
Open Access This is an Open Access article distributed in accordance with the Creative Commons Attribution Non Commercial (CC BY-NC 4.0) license, which permits others to distribute, remix, adapt, build upon this work noncommercially, and license their derivative works on different terms, provided the original work is properly cited and the use is non-commercial. See: http:// creativecommons.org/licenses/by-nc/4.0/

\section{REFERENCES}

1. Greenland S, Finkle WD. A critical look at methods for handling missing covariates in epidemiologic regression analyses. $A m \mathrm{~J}$ Epidemiol 1995;142:1255-64.

2. Sterne JA, White IR, Carlin JB, et al. Multiple imputation for missing data in epidemiological and clinical research: potential and pitfalls. BMJ 2009;338;b2393.

3. White IR, Carlin JB. Bias and efficiency of multiple imputation compared with complete-case analysis for missing covariate values. Stat Med 2010;29:2920-31.

4. Schafer JL, Graham JW. Missing data: our view of the state of the art. Psychol Methods 2002;7:147-77.

5. Simon GA, Simonoff JS. Diagnostic plots for missing data in least squares regression. J Am Stat Assoc 1986;81:501-9.

6. Little RJ. A test of missing completely at random for multivariate data with missing values. J Am Stat Assoc 1988;83:1198-202.

7. Rubin DB. Inference and missing data. Biometrika 1976;63: 581-92.

8. Virtanen TE, Lerkkanen M-K, Poikkeus A-M, et al. Student behavioral engagement as a mediator between teacher, family, and peer support and school truancy. Learn Individual Differences 2014;36:201-6.

9. Mitchell KE, Johnson-Warrington $\mathrm{V}, \mathrm{Apps} \mathrm{LD}$, et al. A selfmanagement programme for COPD: a randomised controlled trial. Eur Respir J 2014;44:1538-47.

10. Jamshidian M, Jalal S, Jansen C. MissMech: An R package for testing homoscedasticity, multivariate normality, and missing completely at random (mCAR). J Stat Software 2014;56:1-30.

11. Janssen KJ, Donders ART, Harrell FE Jr, et al. Missing covariate data in medical research: To impute is better than to ignore. $J$ Clin Epidemiol 2010;63:721-7.

12. Karahalios A, Baglietto L, Carlin JB, et al. A review of the reporting and handling of missing data in cohort studies with repeated assessment of exposure measures. BMC Med Res Methodol 2012;12:96.

13. Karahalios A, Baglietto L, Lee KJ, et al. The impact of missing data on analyses of a time-dependent exposure in a longitudinal cohort: a simulation study. Emerg Themes Epidemiol 2013;10:6.
14. Graham JW. Missing data analysis: making it work in the real world. Annu Rev Psychol 2009;60:549-76.

15. Little RJ. Pattern-mixture models for multivariate incomplete data. J Am Stat Assoc 1993;88:125-34.

16. Hedeker D, Gibbons RD. Application of random-effects pattern-mixture models for missing data in longitudinal studies. Psychol Methods 1997;2:64.

17. Unwin A, Hawkins $\mathrm{G}$, Hofmann $\mathrm{H}$, et al. Interactive graphics for data sets with missing values-mANET. J Computat Graphical Stat 1996;5:113-22.

18. Templ M, Alfons A, Kowarik A, et al. VIM: Visualization and imputation of missing values. $R$ package version 2011;2.

19. Honaker J, King G, Blackwell M. Amelia il: A program for missing data. J Stat Software 2011;45:1-47. http://www.jstatsoft. org/v45/i07

20. Su Y-S, Yajima M, Gelman AE, et al. Multiple imputation with diagnostics (mi) in r: opening windows into the black box. J Stat Software 2011;45:1-31.

21. Cheng X, Cook D, Hofmann H. MissingDataGUI. 2014. http://cran. r-project.org/web/packages/MissingDataGUI/MissingDataGUI.pdf

22. Swayne DF, Buja A. Missing data in interactive high-dimensional data visualization. Comput Stat 1998;13:15-26.

23. James $G$, Witten $D$, Hastie $T$, et al. An introduction to statistical learning. Springer, 2013. http://link.springer.com/content/pdf/10 1007/978-1-4614-7138-7.pdf

24. Breiman L, Friedman J, Stone CJ, et al. Classification and regression trees. CRC press, 1984

25. Hastie T, Tibshirani R, Friedman J. The elements of statistical learning. Springer, 2009. http://link.springer.com/content/pdf/10. 1007/978-0-387-84858-7.pdf

26. Elith J, Leathwick JR, Hastie T. A working guide to boosted regression trees. J Anim Ecol 2008;77:802-13.

27. Therneau TM, Atkinson EJ. An introduction to recursive partitioning using the rpart routine. Technical Report 61, Section of Biostatistics Mayo Clinic, Rochester. 1997. http://www.mayo.edu/research/ documents/biostat-61pdf/doc-10026699

28. Sutton CD. Classification and regression trees, bagging, and boosting. Handb Stat 2005;24:303-29.

29. Friedman JH, Meulman JJ. Multiple additive regression trees with application in epidemiology. Stat Med 2003;22:1365-81.

30. Breiman L. Statistical modeling: The two cultures (with comments and a rejoinder by the author). Stat Sci 2001;16:199-231.

31. R Core Team. R: A Language and Environment for Statistical Computing. Vienna, Austria: R Foundation for Statistical Computing, 2014. http://www.R-project.org/

32. RStudio. RStudio. Boston, MA: RStudio, 2014. http://www.rstudio.org/

33. Ridgeway G. Gbm: Generalized boosted regression models. 2013 http://CRAN.R-project.org/package=gbm 